\title{
Bosonic Szilard Engine Assisted by Feshbach Resonances
}

\author{
J. Bengtsson, ${ }^{1}$ M. Nilsson Tengstrand,${ }^{1}$ and S.M. Reimann ${ }^{1, *}$ \\ ${ }^{1}$ Mathematical Physics and NanoLund, Lund University, Box 118, 22100 Lund, Sweden
}

(Dated: September 26, 2018)

\begin{abstract}
It was recently found that the information-to-work conversion in a quantum Szilard engine can be increased by using a working medium of bosons with attractive interactions. In the original scheme, the work output depends on the insertion and removal position of an impenetrable barrier that acts like a piston, separating the chambers of the engine. Here, we show that the barrier removal process can be made fully reversible, resulting in a full information-to-work conversion if we also allow for the interaction strength to change during the cycle. Hence, it becomes possible to reach the maximum work output per cycle dictated by the second law of thermodynamics. These findings can, for instance, be experimentally verified with ultra-cold atoms as a working medium, where a change of interaction strength can be controlled by Feshbach resonances.
\end{abstract}

\section{INTRODUCTION}

Szilard's famous single-particle engine [1] is the archetype of an information heat engine that extracts work through measurement and feedback operations. The original setup consists of a single particle in a container that is coupled to a single heat bath. Dividing the container into two equally sized parts by a movable piston, the engine employs a type of "Maxwell's demon" that provides the information on which side of the piston the particle resides (measurement). Work may then be extracted from the particle's collisions with the piston that pushes it aside (feedback). The connection between information and work is ensured by the second law of thermodynamics [2-9]: Erasing one bit of information costs at least the entropy $k_{B} \ln 2$, where $k_{B}$ is the Boltzmann constant. Szilard's thought experiment dates back almost a century and has led to a plethora of studies, for example addressing the physicality of the measurement and erasure processes [2-4], or investigating the role of information in thermodynamics in general [9].

A highly interesting question is, how thermodynamic properties are changed when quantum effects are taken into account. Different quantum versions of Szilard's engine have been suggested, with single-particle [10] to many-body working media [11-18]. For engines with non-interacting particles, bosons were found superior to fermions [11]. As first shown for two particles [12] and recently generalized to the many-body regime [18], attractive interactions between the bosons can enhance the information-to-work conversion even further.

Quantum effects in correlated many-particle systems may enhance the performance also of other kinds of quantum heat engines. For instance, it was recently found [19] that a quantum Otto engine [20] with a many-particle working medium consisting of an interacting Bose gas, confined in a time-dependent harmonic trap, is able to outperform a corresponding ensemble of single-particle quantum heat engines.

*reimann@matfys.lth.se

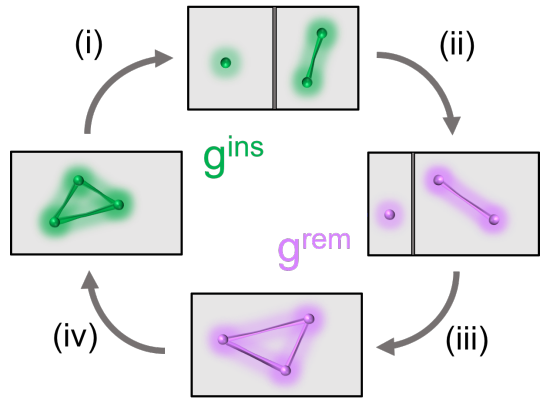

FIG. 1. Schematic picture of a Szilard engine cycle where both the removal position of the barrier and the strength of interactions at barrier insertion and barrier removal, $g^{\text {ins }}$ and $g_{n}^{\text {rem }}$, can be varied. In this particular cycle, one particle was found to the left (and two to the right) of the barrier after insertion.

Szilard-like information-to-work conversion has been experimentally demonstrated for classical systems [2124], but the corresponding setups in the quantum realm $[10-12,16,18]$ have so far evaded realization. However, as noted previously in the literature, see e.g. Refs. [11, 18], such experiments could indeed become possible with ultra-cold atoms. With nowadays quantumoptical trapping techniques, the shape and dimensionality of the confining potential can be modified with a very high degree of experimental control [25], bringing the realization of the quantum Szilard engine closer to reality.

A moving barrier as in the case of the Szilard engine, or a confinement chamber that changes its size as in the Otto cycle, are not the only ways to produce work. Recently it was suggested [26] that a change in the interaction strength, by means of Feshbach resonances [27], can be used to extract work in a bosonic quantum Otto engine. Here, we investigate how a similar concept applies to the bosonic quantum Szilard engine. How does a Feshbach-guided Szilard cycle compare with Szilard's original concept of a moving piston? As a first step, we keep the position of the impenetrable barrier fixed and vary only the strength of the interactions during the cy- 
cle. We then allow for both the interaction strength and the barrier position to vary during the cycle.

To quantify the performance of the many-particle engine, we determine its average work output relative to the value of $k_{B} T \ln 2$ obtained for the original (singleparticle) Szilard engine connected to a heat bath at temperature $T$. We find that for the Feshbach-driven engine, i.e. the engine with fixed barrier position, the maximum relative work output oscillates with the number of bosons, and for larger $N$ indeed exceeds the corresponding Szilard engine with non-interacting bosons and a movable barrier $[11,12]$. Yet, the maximum relative work output, obtained by changing the interaction alone, is smaller than that of an engine with a movable barrier and constant attractive interactions between the bosons [18].

The average work output can be significantly increased if we combine the possibility to tune the interaction strength with the usual Szilard engine setup with a movable wall. In fact, in the deep quantum regime, we find that it is possible to construct a Feshbach-assisted protocol that maximize both the information and the information-to-work conversion efficiency for an arbitrary number of bosons. In other words, by varying the barrier position together with the interaction strength, it is possible to construct an engine that produces the maximum possible work output in the low-temperature limit. The maximal work output is here encountered for working media that undergo a transition between a noninteracting Bose gas and a Tonks-Girardeau gas [28].

The paper is organized as follows: In Sec. II we describe the general quantum Szilard cycle, where both the interactions between the particles and the position of the barrier may be varied in order to maximize the work output. In Sec. III we discuss the work output for the case of a fixed barrier position at the center of the container, where the engine is driven by the variation of the interaction strength alone. Sec. IV then discusses the work output obtained with a simultaneous variation of the interaction strength and the barrier position, i.e. when the conventional Szilard engine is assisted by Feshbach resonances. Finally, in Sec. V, we conclude with prospects for future work.

\section{SZILARD CYCLE FOR BOSONS WITH VARIABLE INTERACTIONS}

For the setup of the Szilard engine, we consider a onedimensional infinite well of length $L$, i.e. a hard-wall box potential with $V(0)=V(L) \rightarrow \infty$ and $V(x)=0$ for $0<x<L$. The trap confines a small number $N$ of spinless bosons interacting by the pseudo-potential of contact type, $g \delta\left(x_{1}-x_{2}\right)$, as commonly used for ultracold atomic gases [25]. Here, $g$ is the strength of the two-body interaction, given in units of $\tilde{g}=\hbar^{2} /(m L)$.

The steps of the Feshbach-assisted quantum manyparticle Szilard cycle are illustrated in Fig. 1: (i) The working medium of $N$ bosonic particles confined in the box, interacting with some initial interaction strength $g=g^{\text {ins }}$, is split into two parts by an impenetrable barrier at a position $x=\ell^{\text {ins }}$; (ii) after separation, the number $n$ of particles on, say, the left side of the wall is measured, and the barrier is then moved to the position $\ell_{n}^{\text {rem }}$, expanding one side of the chamber and compressing the other. In addition, according to the measurement outcome, the interaction strength is changed to $g_{n}^{\mathrm{rem}}$. In step (iii) the barrier is removed, and finally the interaction is tuned back to its initial value $g^{\text {ins }}$ in step (iv).

We assume that all processes are carried out quasistatically and isothermally in contact with a single heat bath. At a given temperature $T$, the change in the partition function

$$
Z=\sum_{j} \mathrm{e}^{-E_{j} /\left(k_{B} T\right)}
$$

determines the work output associated with the isothermal process,

$$
W \leq-\Delta F=k_{B} T \Delta(\ln Z),
$$

where $F$ is the Helmholtz free energy. In Eq. (2), equality is reserved for reversible processes. The sum in Eq. (1) runs over the full spectrum of $N$-body eigenenergies $E_{j}$, which, in turn, depend both on the interaction between the particles and the position of the barrier. For the onedimensional system of bosons with contact interaction, we use the Bethe ansatz [29] to find these energies. For instance, the contribution to $E_{j}$ from the $n_{j}$, where $0 \leq$ $n_{j} \leq N$, bosons located to the left of the barrier at $\ell$ is here given by $\left(\hbar^{2} / 2 m\right) \sum_{\alpha=1}^{n_{j}} k_{(j, \alpha)}^{2}$, where the $k_{(j, \alpha)}$ are the solutions to a set of coupled transcendental equations

$$
\begin{aligned}
\ell k_{(j, \alpha)}= & \pi b_{(j, \alpha)}+\sum_{\beta \neq \alpha} \tan ^{-1}\left(\frac{g m}{\hbar^{2}} \frac{1}{k_{(j, \alpha)}-k_{(j, \beta)}}\right) \\
& +\sum_{\beta \neq \alpha} \tan ^{-1}\left(\frac{g m}{\hbar^{2}} \frac{1}{k_{(j, \alpha)}+k_{(j, \beta)}}\right),
\end{aligned}
$$

The integers $b_{j, \alpha}$ are ordered as $1 \leq b_{(j, 1)} \leq b_{(j, 2)} \leq \cdots \leq$ $b_{\left(j, n_{j}\right)}$. This system of equations can be recast as a nonlinear least squares problem, which we address using a standard trust region algorithm. For $g<0$, the solutions $k_{(j, \alpha)}$ can be complex-valued, which may complicate the numerics (and which effectively limits the negative values of $g^{\text {ins }}$ considered in this work).

The average work output of the full cycle (not accounting for memory processing) of the Feshbach-guided Szilard engine is given by (see Appendix)

$$
W=-k_{B} T \sum_{n=0}^{N} p_{n}\left(\ell^{\mathrm{ins}}, g^{\mathrm{ins}}\right) \ln \left[\frac{p_{n}\left(\ell^{\mathrm{ins}}, g^{\mathrm{ins}}\right)}{p_{n}\left(\ell_{n}^{\mathrm{rem}}, g_{n}^{\mathrm{rem}}\right)}\right],
$$

where $p_{n}(\ell, g)$ is the probability to find $n$ particles to the left of a barrier inserted at $\ell$ when the interaction strength is $g$. All processes except the barrier removal are 
assumed reversible. It should also be noted that for the isothermal processes considered here, the order by which the expansion and interaction tuning steps (ii) are carried out does not matter. This is due to the fact that for reversible isothermal processes, only the initial and final states determine the work of the processes (see Eq. (2)). The probabilities $p_{n}\left(\ell_{n}^{\mathrm{rem}}, g_{n}^{\mathrm{rem}}\right)$ in Eq. (4) characterize the reversibility of the engine [17], and can be thought of as the probabilities to return to a certain configuration if the removal process is performed in reverse. If all $p_{n}\left(\ell_{n}^{\text {rem }}, g_{n}^{\text {rem }}\right)=1$, also the removal process is made reversible and Eq. (4) reduces to $W=k_{B} T I$, where $I=-\sum_{n=0}^{N} p_{n}\left(\ell^{\text {ins }}, g^{\text {ins }}\right) \ln \left[p_{n}\left(\ell^{\text {ins }}, g^{\text {ins }}\right)\right]$ is the Shannon information. This information is maximized by a uniform probability distribution, and the work output of the Szilard engine with $N$ quantum particles is consequently bounded according to $W \leq k_{B} T \ln (N+1)$.

\section{FESHBACH-DRIVEN SZILARD ENGINE}

Let us first consider a bosonic Szilard engine that is driven by a change of the interaction strength alone, with insertion and removal of the barrier at the central position, $\ell^{\text {ins }}=\ell_{n}^{\text {rem }}=L / 2$. This particular choice of barrier position maximizes the Shannon information for non-interacting bosons in the $T \rightarrow 0$ limit. The change in interaction strength from $g^{\text {ins }}$ before insertion to $g_{n}^{\text {rem }}$ at removal of the barrier now plays a role similar to the change in barrier position in the conventional Szilard cycle. For example, let us consider the two-particle engine and cycles in which one boson is measured on either side of the barrier. Simply removing the barrier without any change in $g$, i.e. with $g_{1}^{\text {rem }}=g^{\text {ins }}$, the average work gain equals the average work cost of introducing the barrier in the first place and no net work output is thus possible. If we instead first increase $g$ (which costs no work when only one particle is on either side) such that $g_{1}^{\text {rem }}>g^{\text {ins }}$, the amount of work that can be extracted from removing the barrier is reduced. However, once the barrier is removed, we may extract an additional amount of work by decreasing the interaction strength to its initial value of $g^{\text {ins }}$. In total, the combined work gain is now larger than the cost of introducing the barrier. The positive net work output can, in this case, be explained by the fact that also the losses caused by tunneling particles during the barrier removal process, are reduced when $g_{1}^{\text {rem }}$ is increased.

Depending on the measurement outcome, the values of $g_{n}^{\text {rem }}$ are here chosen from a numerical sweep such that they maximize the relative work output $W /\left(k_{B} T \ln 2\right)$. These maxima are shown in Fig. 2 for different number of bosons and for different choices of $g^{\text {ins }}$. With the exception of $N=2$, the maximal values of $W /\left(k_{B} T \ln 2\right)$ are encountered in the low temperature limit for $g^{\text {ins }}=0$ and at finite temperatures for (finite) $g^{\text {ins }} \neq 0$. The strong odd/even oscillatory behavior in $N$ is in sharp contrast to that of the ordinary Szilard cycle (here shown
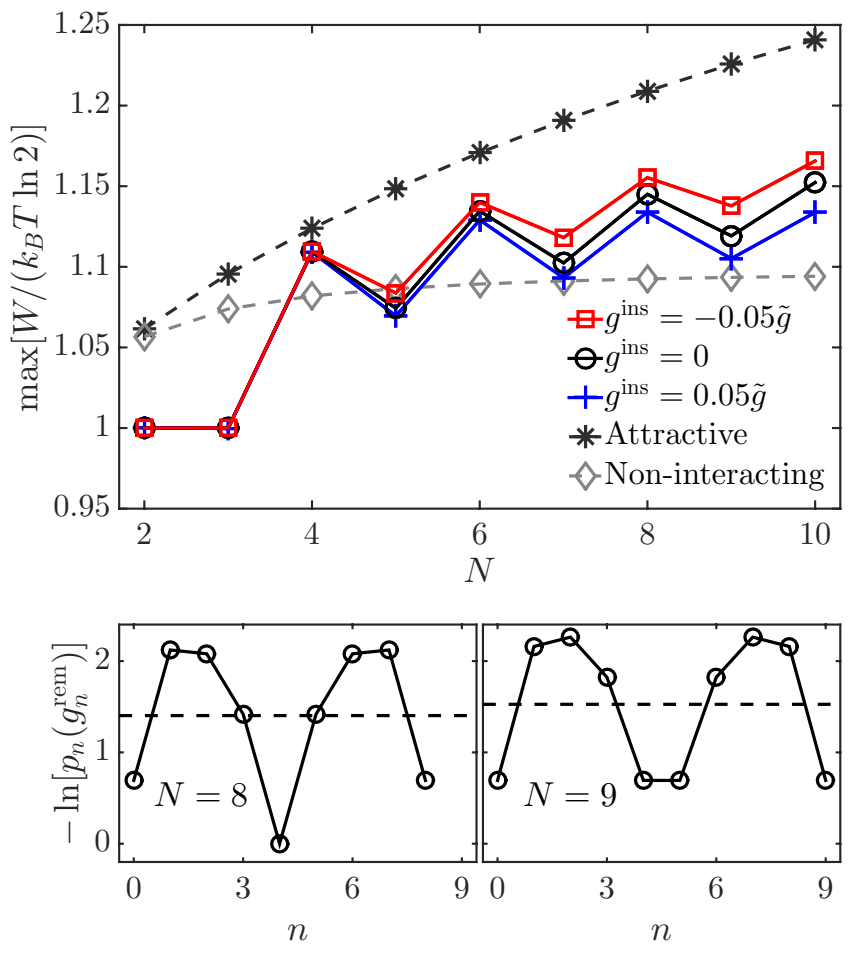

FIG. 2. (Color online) Maximum of the ratio between the average work output $W$ of the Feshbach-driven Szilard engine and the value $k_{B} T \ln 2$, shown for different number of bosons, $N$. The different interaction strengths during the removal of the barrier are optimized to maximize $W$ for each considered $T$. Three different interaction strengths at barrier insertion are considered, namely $g^{\text {ins }}=-0.05 \tilde{g}$ (red line with squares), $g^{\text {ins }}=0$ (black line with circles), and $g^{\text {ins }}=0.05 \tilde{g}$ (blue line with crosses), where $\tilde{g}=\hbar^{2} /(m L)$. For reference we also include the corresponding data for the conventional $N$-particle Szilard engine with a movable barrier. The work output of non-interacting bosons (dashed light grey line with diamonds) is seen together with that of bosons with weak constant attraction (dashed dark grey line with stars). Lower panels: The minimum losses $-\ln \left[p_{n}\left(L / 2, g_{n}^{\text {rem }}\right)\right]$ in the information-to-work conversion for $N=8$ and $N=9$ bosons with $g^{\text {ins }}=0$ and at $k_{B} T=\varepsilon_{1}$, where $\varepsilon_{1}=\hbar^{2} \pi^{2} /\left(2 m L^{2}\right)$ is the single-particle ground state energy in the absence of a barrier. The black dashed lines show the average conversion losses, $-1 /(N+1) \ln \left[p_{n}\left(L / 2, g_{n}^{\text {rem }}\right)\right]$.

for reference as grey dashed lines in Fig. 2, both for the non-interacting [11] and the weakly interacting [18] case), which shows a smooth increase in $W /\left(k_{B} T \ln 2\right)$ with $N$.

To understand the origin to the oscillatory $N$-behavior seen in Fig. 2, a closer examination of the probabilities $p_{n}\left(L / 2, g_{n}^{\text {rem }}\right)$ is called for. The two probabilities $p_{0}\left(L / 2, g_{0}^{\mathrm{rem}}\right)$ and $p_{N}\left(L / 2, g_{N}^{\mathrm{rem}}\right)$ are maximized in the limit of a strong attractive interaction

$$
p_{0}\left(L / 2, g_{0}^{\mathrm{rem}} \rightarrow-\infty\right)=p_{N}\left(L / 2, g_{N}^{\mathrm{rem}} \rightarrow-\infty\right) \rightarrow 1 / 2 .
$$

Contrary, the limit of a strong repulsive interaction max- 
imizes the probability for a configuration with an equal, or almost equal, number of particles on either side of the barrier,

$$
\begin{aligned}
p_{N / 2}\left(L / 2, g_{N / 2}^{\text {rem }} \rightarrow \infty\right) & \rightarrow 1, \quad \text { for even } N \\
p_{(N \pm 1) / 2}\left(L / 2, g_{(N \pm 1) / 2}^{\text {rem }} \rightarrow \infty\right) \rightarrow 1 / 2, & \text { for odd } N .
\end{aligned}
$$

The central features in the optimal probability distribution, associated with the barrier removal, are thus slightly different for an even and for an odd number of particles, with a fully reversible removal process possible for the former. In Fig. 2, we show the corresponding minimum losses, $-\ln p_{n}\left(L / 2, g_{n}^{\text {rem }}\right)$, associated with the information-to-work conversion and the average value $-1 /(N+1) \sum_{n} p_{n}\left(L / 2, g_{n}^{\text {rem }}\right)$ for $N=8$ and $N=9$ bosons at $k_{B} T=\varepsilon_{1}$ (which is sufficiently low for $p_{n}(L / 2,0)$ to be approximately uniformly distributed) when $g^{\text {ins }}=0$. Here $\varepsilon_{i}=\hbar^{2} \pi^{2} i^{2} /\left(2 m L^{2}\right)$ is the singleparticle energy in the absence of a barrier. We see that the average conversion loss is higher for $N=9$ than for $N=8$. Even though the information grows like $\ln (N+1)$ in the low-temperature-limit, the increase in conversion losses, going from an even number $N$ of particles to $N+1$, turns out to be large enough for the total work to decrease. If we instead add two particles, keeping the number of particles odd or even, the increase in information dominates over the increase in losses. The general overall trend is thus that the maximum in $W /\left(k_{B} T \ln 2\right)$ increases with $N$, but with an oscillatory odd/even modification.

For $N \geq 4$, we also observe (see Fig. 2) that a higher work ratio, $W /\left(k_{B} T \ln 2\right)$, may be achieved for bosons with attractive interactions, compared to non-interacting ones, during the barrier insertion. In general, in the search for the maximize in $W /\left(k_{B} T\right)$ it is important to account for the losses in the information-to-work conversion. The maximum in $W /\left(k_{B} T\right)$ is thus not necessarily seen at the maximum Shannon information. In other words, the fact that $I$ is maximal for non-interacting bosons in the zero temperature limit does not guarantee that also $W /\left(k_{B} T\right)$ is the highest possible with this particular setup. For attractive bosons, the higher values of $W /\left(k_{B} T\right)$ are instead obtained at finite temperatures where both the information-to-work conversion efficiency and the Shannon information are relatively high (but neither of them are maximal), see Fig. 3 for an engine with $N=5$. Similar features have also been seen in the conventional $N$-particle Szilard engine with a movable barrier, as further discussed in Ref. [18].

Let us finally investigate the cases of $N=2$ and $N=3$, where the attractive interaction does not seem to increase the maxima in $W /\left(k_{B} T \ln 2\right)$. With a central barrier insertion position, $p_{n}\left(L / 2, g^{\text {ins }}\right)=p_{N-n}\left(L / 2, g^{\text {ins }}\right)$ for $n=0, \ldots, N$. This symmetry in $p_{n}\left(L / 2, g^{\text {ins }}\right)$, combined with the fact that $\sum_{n} p_{n}\left(L / 2, g^{\text {ins }}\right)=1$, allows us to express the optimal average work output per cycle as
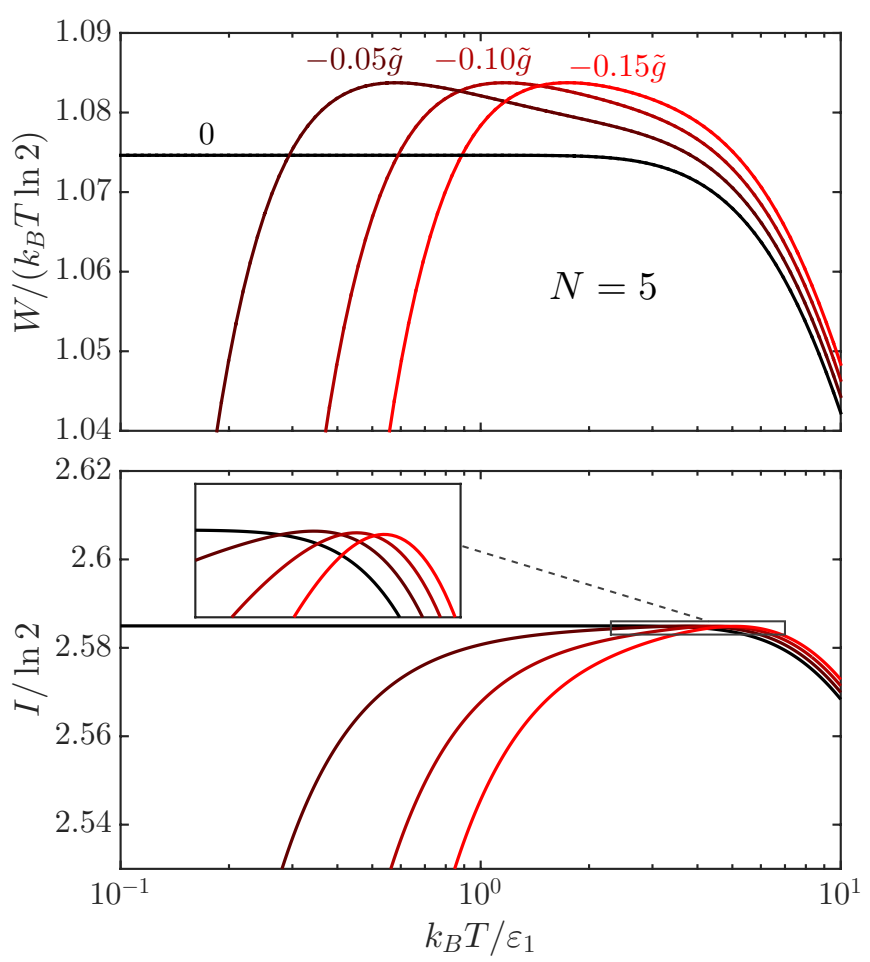

FIG. 3. (Color online) Upper panel: Work output of the Feshbach-driven Szilard engine for $N=5$ as a function of temperature and for different interaction strength $g^{\text {ins }}$. Lower panel: Information $I=-\sum_{n} p_{n}\left(L / 2, g^{\text {ins }}\right)$ as a function of temperature for the same systems. For a fully reversible cycle, $W=k_{B} T I$.

a function of $p_{0}\left(L / 2, g^{\text {ins }}\right)$ alone,

$$
\begin{aligned}
W= & -k_{B} T\left\{2 p_{0}\left(L / 2, g^{\mathrm{ins}}\right) \ln \left[2 p_{0}\left(L / 2, g^{\mathrm{ins}}\right)\right]\right. \\
& \left.+\left[1-2 p_{0}\left(L / 2, g^{\mathrm{ins}}\right)\right] \ln \left[1-2 p_{0}\left(L / 2, g^{\mathrm{ins}}\right)\right]\right\},
\end{aligned}
$$

where the probabilities in Eqs. $(5,6,7)$ are used to maximize $W$. The work output in Eq. (8) has a peak value of $W=k_{B} T \ln 2$, which is obtained for $p_{0}\left(L / 2, g^{\mathrm{ins}}\right)=1 / 4$, see Fig. 4. For $N=3$, the largest ratio $W /\left(k_{B} T \ln 2\right)$ is consequently retrieved with a uniform probability distribution $p_{n}\left(L / 2, g^{\text {ins }}\right)$, i.e. with a maximal Shannon information. Such a probability distribution is, in turn, obtained in the $T \rightarrow 0$ limit when $g^{\text {ins }}=$ 0 . For $g^{\text {ins }}<0$, the same optimal probability distribution, and thus maximum in $W /\left(k_{B} T \ln 2\right)$, is instead seen at a finite temperature. If we start in the low-temperature limit (where $p_{0}\left(L / 2, g^{\text {ins }}\right)=1 / 2$ ) and continuously increase $T$, then we will eventually pass through the optimal value $p_{0}\left(L / 2, g^{\text {ins }}\right)=1 / 4$ towards $p_{0}\left(L / 2, g^{\text {ins }}\right)=1 / 8$ in the classical limit $(T \rightarrow \infty)$. For $N=2$, the maximum in $W /\left(k_{B} T \ln 2\right)$, is obtained for $p_{1}\left(L / 2, g^{\text {ins }}\right)=2 p_{0}\left(L / 2, g^{\text {ins }}\right)$, and thus not at the maximal Shannon information. Regardless of the (finite) in- 


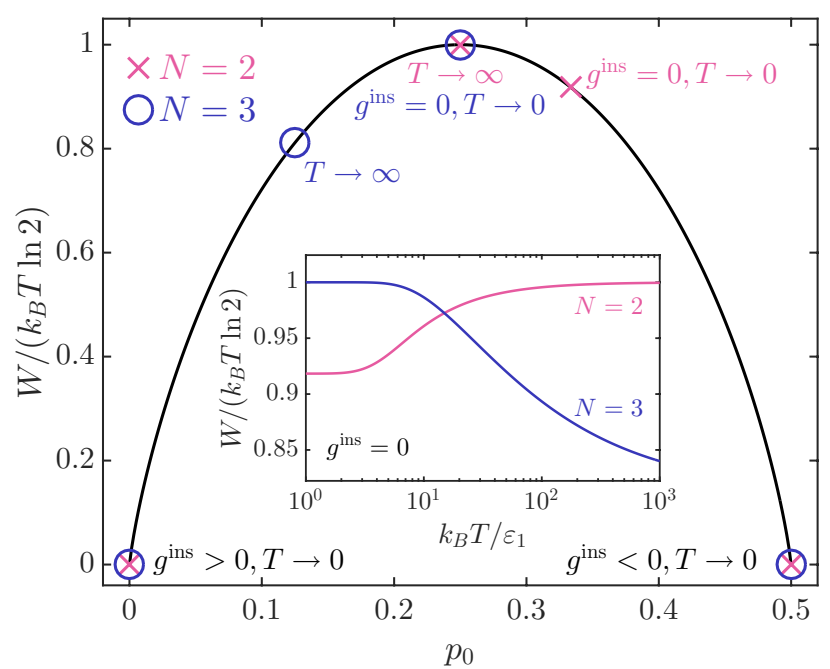

FIG. 4. (Color online) Work output of the Feshbach-driven Szilard engine for $N=2$ and $N=3$ as a function of the probability $p_{0}\left(L / 2, g^{\text {ins }}\right)$ to find zero particles to the left of the barrier after insertion. Inset: Work output $W /\left(k_{B} T \ln 2\right)$ for $N=2$ and $N=3$ as a function of temperature when $g^{\text {ins }}=0 . \quad \varepsilon_{1}=\hbar^{2} \pi^{2} /\left(2 m L^{2}\right)$ is the single-particle ground state energy in the absence of a barrier.

teraction strength, for two bosons, we approach the optimal value $p_{0}\left(L / 2, g^{\text {ins }}\right)=1 / 4$ in the classical limit.

\section{FESHBACH-ASSISTED SZILARD ENGINE}

Let us now incorporate the interaction-tuning step into the conventional cycle of the many-particle quantum Szilard engine $[11,12,18]$. In other words, in addition to choosing $g_{n}^{\text {rem }}$ optimally to maximize the work output we now also simultaneously choose the optimal values for the removal positions $\ell_{n}^{\text {rem }}$. We consider a central insertion position, i.e. $\ell^{\text {ins }}=L / 2$, which was found optimal in the region of largest $W /\left(k_{B} T \ln 2\right)$ for non-interacting and attractively interacting bosons in the conventional Szilard engine [18]. The work output of the Feshbach-assisted Szilard engine with $N \leq 8$ bosons is shown in Fig. 5 . Note that we here show the ratio $W /\left[k_{B} T \ln (N+1)\right]$ as a function of $T$. Of key interest is that the maximal possible average work output, only bounded by the second law for feedback processes [9], can be achieved at low temperatures for $g^{\text {ins }}=0$. First, we recall that the Shannon information is maximal for bosons in the $T \rightarrow 0$ limit when $\ell^{\text {ins }}=L / 2$ and $g^{\text {ins }}=0$. Secondly, the full information-to-work conversion may be explained by the fact that in a one-dimensional system, bosons with infinitely strong repulsive contact-interactions act as spinpolarized fermions, i.e., a Tonks-Girardeau [28] gas. In other words, when $g_{n}^{\text {rem }} \rightarrow \infty$ for all $n=0, \ldots, N$, the fermionized bosons fill up the single-particle energy levels according to the Pauli exclusion principle. A barrier

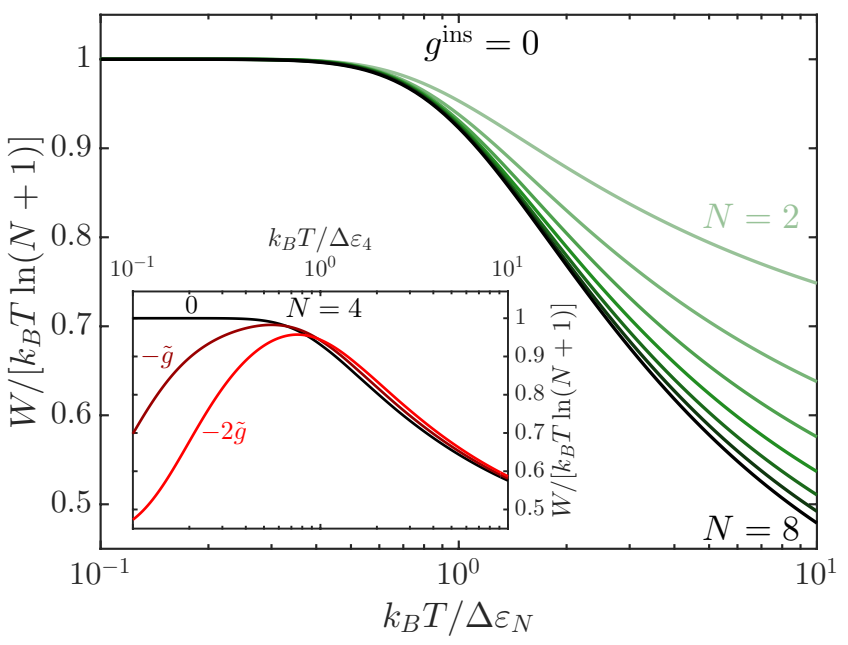

FIG. 5. (Color online) Relative work output, $W /\left[k_{B} T \ln (N+\right.$ $1)$ ], of the Feshbach-assisted Szilard engine as a function of temperature $T$ for $N \leq 8$ particles, with $\Delta \varepsilon_{N}=\epsilon_{N+1}-\epsilon_{N}$. The different lines are for different particle numbers, from $N=2$ (top) to $N=8$ (bottom). Inset: Effect of different interaction strengths $g^{\text {ins }}$ at insertion (as indicated in the figure) for the example of $N=4$ particles in the engine. The relative work output is significantly lowered in the deep quantum regime, but increases at higher temperature for an initial attraction.

position may then always be found such that the manyparticle ground state consists of $n$ particles to the left of the barrier and $N-n$ to the right. In the considered lowtemperature limit, the removal process can thus always be made fully reversible with a complete information-towork conversion. A straightforward calculation reveals the intervals for optimal removal,

$$
\frac{n}{N+1}<\frac{\ell_{n}^{\text {rem }}}{L}<\frac{n+1}{N+1} .
$$

Also with $g^{\text {ins }}<0$, a maximal information-to-work conversion efficiency is possible in the low-temperature limit. However, since all the particles necessarily are found on the same side of the barrier upon measurement, the Shannon information is drastically reduced and, as a consequence, also the work output, see the inset of Fig. 5.

If we increase the temperature, the work output can be seen to decrease when $g^{\text {ins }}=0$ (see Fig. 5). This degradation of the engine is of twofold origin: Partly, it occurs due to a reduction in the Shannon information, and partly it is because of a loss of reversibility associated with the barrier removal process. With increased temperature, the distribution of measurement outcomes goes from $p_{n}(L / 2,0) \rightarrow 1 /(N+1)$ (in the $T \rightarrow 0$ limit) to the classical distribution where the particles behave as distinguishable ones, $p_{n}(L / 2,0) \rightarrow\left(\begin{array}{c}N \\ n\end{array}\right) / 2^{N}$ (in the $T \rightarrow \infty$ limit). The latter distribution is peaked about $n \sim N / 2$ and thus has a lower Shannon information. In the case of $g^{\text {ins }}<0$, on the other hand, the information will typically grow with $T$ initially, when more measure- 

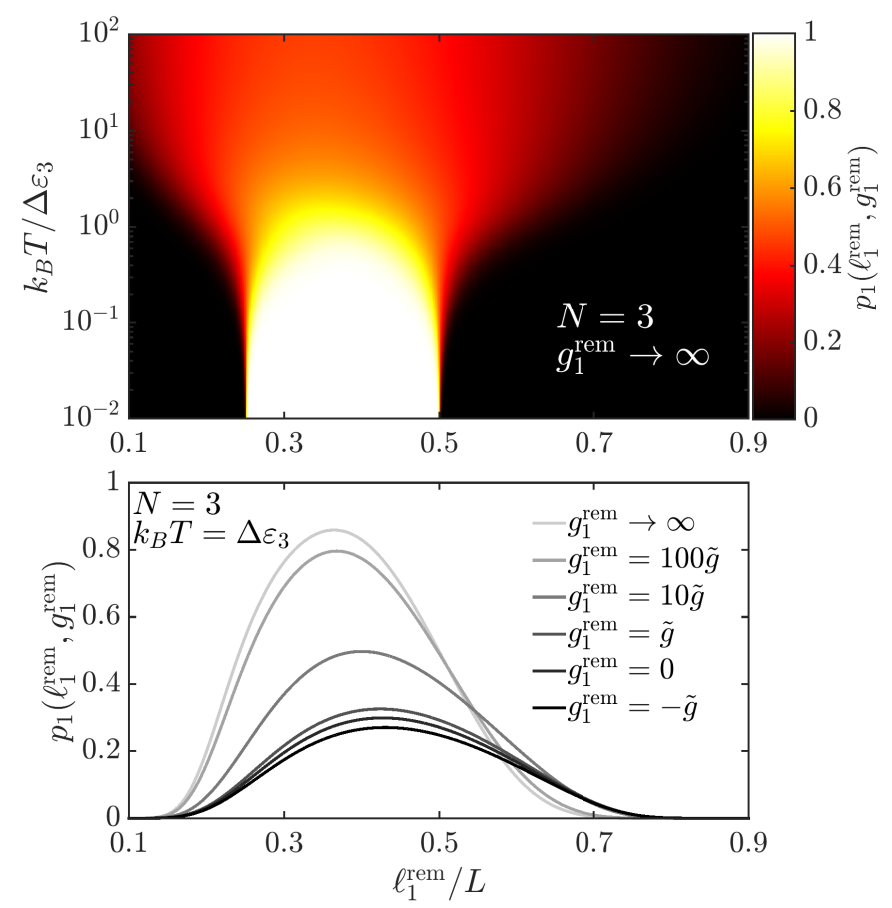

FIG. 6. (Color online) Upper panel: The reversibility probability $p_{1}\left(\ell_{1}^{\mathrm{rem}}, g_{1}^{\mathrm{rem}}\right)$ as a function of the removal position $\ell_{1}^{\text {rem }}$ and temperature $T$ at $g_{1}^{\text {rem }} \rightarrow \infty$, i.e., at complete fermionization, for the case of $N=3$ particles. Lower panel: $p_{1}\left(\ell_{1}^{\mathrm{rem}}, g_{1}^{\mathrm{rem}}\right)$ as a function of $\ell_{1}^{\mathrm{rem}}$ at $k_{B} T / \Delta \varepsilon_{3}=1$ for different values of $g_{1}^{\text {rem }}$. We can see that optimal removal still occurs at complete fermionization even though the removal process is no longer reversible.

ment outcomes becomes accessible, but will later decay as the classical limit is approached. Similar to the work output of the Feshbach-driven engine seen in Fig. 3, the ratio $W /\left[k_{B} T \ln (N+1)\right]$ now has a maximum (although typically smaller than the maxima for $g^{\text {ins }}=0$ ) at a finite temperature (see the inset of Fig. 5). Furthermore, also similar to the Feshbach-driven engine, it is important to also account for the information-to-work conversion losses when establishing the details of this maximum.

The second reason for the reduction in $W /\left[k_{B} T \ln (N+\right.$ $1)$ ], namely the growing losses in the information-to-work conversion with $T$, is illustrated for the case of $N=3$ in Fig. 6. In particular, we consider the case in which one boson is found to the left of the barrier and show the probability $p_{1}\left(\ell_{1}^{\text {rem }}, g_{1}^{\text {rem }} \rightarrow \infty\right)$ as a function of the barrier removal position. Clearly, in the limit of $T \rightarrow 0$, a fully reversible removal process is possible, in agreement with the condition in Eq. (9). As the temperature increases, excited many-body states starts to become more populated during the barrier removal process. The probability $p_{1}\left(\ell_{1}^{\text {rem }}, g_{1}^{\text {rem }} \rightarrow \infty\right)$ will then decrease, indicating growing losses in the information-to-work conversion. We may finally estimate the onset of these conversion losses based on that the thermal energy has to be of the or- der of $k_{B} T \sim \Delta \varepsilon_{N}=\varepsilon_{N+1}-\varepsilon_{N}=(N+1)^{2} \varepsilon_{1}-N^{2} \varepsilon_{1}=$ $(2 N+1) \varepsilon_{1}$ for any excited many-body state to have a significant population. Note that the temperatures shown in Fig. 6, as well as in Fig. 5, are scaled by the factor $\Delta \epsilon_{N}$.

\section{CONCLUSION}

This work suggests an optimal protocol for the quantum Szilard engine, where the interaction strength of the working medium is allowed to vary according to the measurement outcome. We have shown that by adding this new aspect to the regular scheme of the Szilard engine, it can be made fully reversible in the quantum regime and reach the maximal possible work output per cycle as dictated by the second law of thermodynamics. For a sufficiently strong repulsion between the quantum particles constituting the engine's medium and at low enough temperatures, there exists an interval of values for each removal position such that the engine is made reversible. Furthermore, we have seen that deterioration in work output at higher temperatures can be decreased by improving the engine's information content through the addition of an initial attraction to the working medium. The setup suggested here could be realizable for example with ultra-cold atoms where the interactions can be controlled by Feshbach resonances. Our work opens important new perspectives for information-driven quantum heat engines, for which the concept of the Szilard cycle since long has been the prime paradigm.

\section{ACKNOWLEDGMENTS}

We thank G. Kiršanskas for very valuable comments and his help regarding the implementation of the Bethe ansatz. We also thank T. Busch, H. Linke, P. Samuelsson, M. Ueda and A. Wacker for many helpful discussions on the many-body Szilard engine. This work was financially supported by Knut and Alice Wallenberg Foundation, NanoLund and the Swedish Research Council.

\section{Appendix: Derivation of Work Expression}

For the derivation for the work expression for the Feshbach-assisted Szilard engine, we follow very closely the concept suggested by Kim et al. [11] that was also applied in Ref. [18]. The average work output associated with a change of the length and interaction strength parameters from some initial values $\left(\ell_{i}, g_{i}\right)$ to some final values $\left(\ell_{f}, g_{f}\right)$ in an isothermal process is given by

$$
W_{\text {iso }}=k_{B} T \ln \left[\frac{Z\left(\ell_{f}, g_{f}\right)}{Z\left(\ell_{i}, g_{i}\right)}\right]
$$


where $Z=\sum_{n} e^{-E_{n} /\left(k_{B} T\right)}$ is the partition function. In the first step of the cycle, (i), a barrier is raised at $\ell^{\text {ins }}$ for $N$ particles with interaction strength $g^{\text {ins }}$, which costs an amount of work equal to

$$
W_{(i)}=k_{B} T \ln \left[\frac{\sum_{n=0}^{N} Z_{n}\left(\ell^{\mathrm{ins}}, g^{\mathrm{ins}}\right)}{Z_{N}\left(L, g^{\mathrm{ins}}\right)}\right] .
$$

Here $Z_{n}(\ell, g)$ is the partition function obtained when the sum runs over the energies with $n$ particles to the left of the barrier alone. In the second step, the number of particles on each side is measured and subsequently an expansion is performed until the barrier reaches $\ell_{n}^{\text {rem }}$. The work associated with this expansion/compression step of the cycle is given by

$$
W_{(i i)}^{(1)}=k_{B} T \sum_{n=0}^{N} p_{n}\left(\ell^{\text {ins }}, g^{\text {ins }}\right) \ln \left[\frac{Z_{n}\left(\ell_{n}^{\text {rem }}, g^{\text {ins }}\right)}{Z_{n}\left(\ell^{\text {ins }}, g^{\text {ins }}\right)}\right],
$$

where $p_{n}=Z_{n} / \sum_{m=0}^{N} Z_{m}$ is the probability of measuring $\mathrm{n}$ particles to the left of the barrier.

Furthermore, the interaction strength is changed to $g_{n}^{\text {rem }}$ depending on the measurement outcome, giving the additional work

$$
W_{(i i)}^{(2)}=k_{B} T \sum_{n=0}^{N} p_{n}\left(\ell^{\text {ins }}, g^{\text {ins }}\right) \ln \left[\frac{Z_{n}\left(\ell_{n}^{\text {rem }}, g_{n}^{\text {rem }}\right)}{Z_{n}\left(\ell_{n}^{\text {rem }}, g^{\text {ins }}\right)}\right] .
$$

Next, in step (iii), the barrier is removed, and as its height is lowered, particles will eventually be able to tunnel between the two systems. Provided that the processes are carried out quasi-statically, the average work associated with this process reads (see Ref. [11])

$$
W_{(i i i)}=k_{B} T \sum_{n=0}^{N} p_{n}\left(\ell^{\mathrm{ins}}, g^{\mathrm{ins}}\right) \ln \left[\frac{Z_{N}\left(L, g_{n}^{\mathrm{rem}}\right)}{\sum_{n=0}^{N} Z_{n}\left(\ell_{n}^{\text {rem }}, g_{n}^{\text {rem }}\right)}\right] \text {. }
$$

In the fourth and last step, the interaction strength is changed back to its original value, from $g_{n}^{\text {rem }}$ to $g^{\text {ins }}$, and

$$
W_{(i v)}=k_{B} T \sum_{n=0}^{N} p_{n}\left(\ell^{\mathrm{ins}}, g^{\mathrm{ins}}\right) \ln \left[\frac{Z_{N}\left(L, g^{\mathrm{ins}}\right)}{Z_{N}\left(L, g_{n}^{\text {rem }}\right)}\right] .
$$

Finally we get the total average work output per cycle by summing up the different contributions $W=W_{(i)}+$ $W_{(i i)}^{(1)}+W_{(i i)}^{(2)}+W_{(i i i)}+W_{(i v)}$.
[1] L. Szilard, Z. Phys. 53, 840 (1929).

[2] R. Landauer, IBM J. Res. Dev. 5, 183 (1961).

[3] C. H. Bennett, International Journal of Theoretical Physics 21, 905 (1982).

[4] C. H. Bennett, Studies in History and Philosophy of Science Part B: Studies in History and Philosophy of Modern Physics 34, 501 (2003), quantum Information and Computation.

[5] H. S. Leff and A. F. Rex, Maxwell's Demon 2: Entropy, Classical and Quantum Information Computing (IOP Publishing, Bristol, 2003).

[6] M. Plenio and V. Vitelli, Contemp. Phys. 42, 25 (2001).

[7] T. Sagawa and M. Ueda, Phys. Rev. Lett. 100, 080403 (2008).

[8] T. Sagawa and M. Ueda, Phys. Rev. Lett. 102, 250602 (2009).

[9] J. M. R. Parrondo, J. M. Horowitz, and T. Sagawa, Nat Phys 11, 131 (2015).

[10] W. H. Zurek, in Frontiers of Nonequilibrium Statistical Physics, edited by G. T. Moore and M. O. Scully (Springer US, Boston, MA, 1986) pp. 151-161.

[11] S. W. Kim, T. Sagawa, S. De Liberato, and M. Ueda, Phys. Rev. Lett. 106, 070401 (2011).

[12] K.-H. Kim and S. W. Kim, J. Korean Phys. Soc. 61, 1187 (2012).

[13] C. Y. Cai, H. Dong, and C. P. Sun, Phys. Rev. E 85, 031114 (2012).

[14] Y. Lu and G. L. Long, Phys. Rev. E 85, 011125 (2012).

[15] Z. Zhuang and S.-D. Liang, Phys. Rev. E 90, 052117
(2014).

[16] M. Plesch, O. Dahlsten, J. Goold, and V. Vedral, Sci. Rep. 4, 6995 (2014).

[17] H. J. Jeon and S. W. Kim, New J. Phys. 18, 043002 (2016).

[18] J. Bengtsson, M. Nilsson Tengstrand, A. Wacker, P. Samuelsson, M. Ueda, H. Linke, and S. M. Reimann, Phys. Rev. Lett. 120, 100601 (2018).

[19] J. Jaramillo, M. Beau, and A. del Campo, New Journal of Physics 18, 075019 (2016).

[20] H. T. Quan, Y.-x. Liu, C. P. Sun, and F. Nori, Phys. Rev. E 76, 031105 (2007).

[21] S. Toyabe, T. Sagawa, M. Ueda, E. Muneyuki, and M. Sano, Nature Physics 6, 988 (2010).

[22] E. Roldan, I. A. Martinez, J. M. R. Parrondo, and D. Petrov, Nat Phys 10, 457 (2014).

[23] J. V. Koski, V. F. Maisi, J. P. Pekola, and D. V. Averin, Proc. Natl. Acad. Sci. 111, 13786 (2014).

[24] J. V. Koski, A. Kutvonen, I. M. Khaymovich, T. AlaNissila, and J. P. Pekola, Phys. Rev. Lett. 115, 260602 (2015).

[25] I. Bloch, J. Dalibard, and W. Zwerger, Rev. Mod. Phys. 80, 885 (2008).

[26] J. Li, T. Fogarty, S. Campbell, X. Chen, and T. Busch, New Journal of Physics 20, 015005 (2018).

[27] C. Chin, R. Grimm, P. Julienne, and E. Tiesinga, Reviews of Modern Physics 82, 1225 (2010).

[28] M. Girardeau, J. Math. Phys. 1, 516 (1960).

[29] M. Gaudin, Phys. Rev. A 4, 386 (1971). 